\title{
Instrumental systematics and weak gravitational lensing
}

\author{
Rachel Mandelbaum ${ }^{a}$ \\ ${ }^{a}$ McWilliams Center for Cosmology, Department of Physics, Carnegie Mellon University \\ 5000 Forbes Ave., Pittsburgh, PA 15213, USA \\ E-mail: rmandelbeandrew.cmu.edu
}

\begin{abstract}
We present a pedagogical review of the weak gravitational lensing measurement process and its connection to major scientific questions such as dark matter and dark energy. Then we describe common ways of parametrizing systematic errors and understanding how they affect weak lensing measurements. Finally, we discuss several instrumental systematics and how they fit into this context, and conclude with some future perspective on how progress can be made in understanding the impact of instrumental systematics on weak lensing measurements.
\end{abstract}

KEYWORDS: Data analysis; Image processing; Photon detectors for UV, visible and IR photons. 


\section{Contents}

1. Motivation 囵

2. Scientific background 2

2.1] Weak lensing measurements 2]

2.2 The impact of systematics in shear estimation 3

3. Instrumental systematics 6

3.1 Brighter-fatter effect

3.2 Nonlinearity

4. Conclusions

\section{Motivation}

In our current cosmological model, only $4 \%$ of the Universe consists of the baryonic matter from which stars and planets are made. To explain a wide variety of cosmological observations, we have been forced to posit the existence of dark matter (detected through its gravitational attraction) and dark energy (which causes a repulsion that is driving the accelerated expansion of the Universe). While we infer the existence of these dark components, the question of what they actually are remains a mystery.

Gravitational lensing [1, 2], 3] is the deflection of light from distant objects by the matter along its path to us - all of the matter, including dark matter. Lensing measurements are thus directly sensitive to dark matter. They also allow us to infer the properties of dark energy [ [由], because the accelerated expansion of the Universe that it causes directly opposes the effects of gravity, which tends to cause matter to clump into ever larger structures. This measurement relies on the small but spatially coherent distortions (known as weak shears) in the shapes of distant galaxies, which provide a statistical map of cosmological large-scale structure along the line-of-sight. A key problem for weak lensing measurement is that there are many other effects in astronomical images that cause coherent distortions of galaxy shapes and that must be effectively removed in order to reliably measure weak lensing.

Because of its sensitivity to dark matter and dark energy, ever-larger surveys have been planned to measure weak lensing increasingly precisely, including Euclid ${ }^{1}$ [[]], LSST $^{2}$ [6], and WFIRSTAFTA $^{3}[$ [Z], all of which are Stage IV dark energy experiments according to the Dark Energy Task

\footnotetext{
${ }^{1}$ http://sci.esa.int/euclid/, http://www.euclid-ec.org

${ }^{2}$ http: //www.lsst.org/lsst/

${ }^{3}$ http: //wfirst.gsfc.nasa.gov
} 
Force [8] definitions. The very high signal-to-noise ratio of the weak lensing measurements in these surveys will necessitate excellent understanding and control of systematic uncertainties.

Broadly speaking, among the sources of systematic uncertainty in weak lensing measurements are (1) instrumental systematics, (2) the need to remove the point-spread function (PSF) which blurs the galaxy images to robustly infer the lensing shear, (3) the need to estimate redshifts for the galaxies or at least statistically understand their distribution, and (4) theoretical uncertainties such as the impact of baryons on the measured quantities (usually predicted using dark-matter-only simulations) and failures in the assumption that galaxy shapes are randomly oriented in the absence of lensing. Of these, issues (2) through (4) have been thoroughly explored in the literature (see, for example, [1, 10, 11, 112, 13, 14, 15, 16, 17]] and references therein). Instrumental systematics have received somewhat less attention, and are the main topic of discussion in this pedagogical review.

We begin in section 2 by discussing how weak lensing measurements are made, and how those measurements are affected by systematic errors in general. In section 3 we discuss instrumental systematics more specifically. We conclude in section $\$$ with future outlook.

\section{Scientific background}

\subsection{Weak lensing measurements}

In this subsection, we ignore the question of systematics, and give a basic summary of how weak gravitational lensing measurements are carried out.

Cosmic shear, the measurement of weak lensing by large-scale structure, relies on correlating pairs of galaxy shapes as a function of their separation on the sky. Galaxies that are nearby on the sky tend to have correlated shapes due to having been lensed by associated structures that subject them to similar weak lensing shears. Galaxies that are farther apart on the sky have much less correlated weak lensing shears.

To carry out this measurement, the most basic requirement is a catalog of galaxy positions and their shape estimates ${ }^{4}$, which we take as a proxy for shear (but see section 2.2 for more details on the difference between galaxy shapes and shear). While galaxy light profiles do not in general have elliptical isophotes, they are nonetheless commonly described in terms of some effective ellipticity magnitude $|\varepsilon|$, which can be defined in terms of the minor-to-major axis ratio, and position angle $\phi$ with respect to some coordinate system on the sky. Alternatively, one can write two components of the ellipticity as $\varepsilon_{1}=|\varepsilon| \cos 2 \phi, \varepsilon_{2}=|\varepsilon| \sin 2 \phi$, and define a complex ellipticity as $\varepsilon=\varepsilon_{1}+$ $\mathrm{i} \varepsilon_{2}$. These trigonometric functions of $2 \phi$ are very common in mathematical expressions related to galaxy shapes or lensing shears, due to their being spin-2 quantities (ellipses are invariant under $180^{\circ}$ rotations). For more details on common definitions of galaxy shapes, see for example [19].

The galaxy shapes will have some large random intrinsic components which is a key source of statistical error in most weak lensing measurements, along with a small but fortunately coherent weak lensing shear. The intrinsic shape then averages out of correlation functions of galaxy shapes,

\footnotetext{
${ }^{4}$ Technically, one need not have a per-galaxy shape or shear estimate to reconstruct the lensing shear field; see [18] for options that avoid calculating a per-object shear in favor of ensemble estimates. However, these methods are relatively new and their use for cosmic shear requires further practical and theoretical development, so for the remainder of this review we assume that weak lensing measurements will use per-object galaxy shapes.
} 
while the lensing shear does not (but see [17] for issues related to this basic assumption). An estimate of the galaxy redshift distribution is also needed in order to make a theoretical prediction for the cosmic shear correlations. However, even more promising is the so-called cosmic shear tomography, which involves dividing the galaxies into redshift bins based on a per-galaxy photometric redshift estimate (see, for example, figure 1 in [20]). With $>1$ redshift bin, additional information can be obtained by correlating galaxies both within and across those redshift slices, and seeing how the shear signal changes in each case.

A typical cosmic shear correlation function estimator, for the correlation function corresponding to redshift slices $i$ and $j$ is [20]

$$
\hat{\xi}_{ \pm}^{i j}(\theta)=\frac{\sum w_{a} w_{b}\left[\varepsilon_{t}^{i}\left(\mathbf{x}_{a}\right) \varepsilon_{t}^{i}\left(\mathbf{x}_{b}\right) \pm \varepsilon_{\times}^{i}\left(\mathbf{x}_{a}\right) \varepsilon_{\times}^{i}\left(\mathbf{x}_{b}\right)\right]}{\sum w_{a} w_{b}} .
$$

The index $a$ runs over all galaxies in redshift slice $i$, while the index $b$ runs over all galaxies in redshift slice $j$. Galaxy pairs $(a, b)$ are identified, and their angular separation on the sky $\theta$ (calculated using their positions $\mathbf{x}_{a, b}$ ) is used to put them into bins in $\theta$. Each galaxy has a weight factor $w_{a, b}$ that relates to uncertainty in the shear estimates. Each galaxy's shear estimate $\varepsilon$, which has two components, is rotated into a coordinate system defined by the vector connecting galaxies $a$ and $b$. The component corresponding to orientation along or at $90^{\circ}$ with respect to that vector is $\varepsilon_{t}$, and the component corresponding to orientation along the $\pm 45^{\circ}$ rotated system is $\varepsilon_{\times}$. The two estimated correlation functions for each pair of redshift bins $i$ and $j, \hat{\xi}_{ \pm}^{i j}(\theta)$, both relate to integrals over the matter power spectrum along the line-of-sight. For examples of recent cosmic shear measurements using several different datasets, see [20, 21, 22, 23]. These have been used to constrain the amplitude of the matter power spectrum and its evolution with time, which tells us about the equation of state of dark energy.

Another type of weak lensing measurement is cluster-galaxy or galaxy-galaxy lensing, which involves identifying specific foreground galaxies or clusters that act as "lenses", and correlating them with the positions of background sources. This measurement is therefore a (lens) position vs. (source) shape cross-correlation function, which makes it less subject to certain systematic errors in shear estimates. It provides an estimate of the projected total matter surface density around the chosen lenses. While it has primarily been used to make the connection between galaxies or clusters and their host dark matter halos (e.g., very recently, [24, 25]), it can also be used in combination with estimates of galaxy clustering to constrain the growth of cosmic structure and therefore dark energy [26], and to test whether General Relativity is the effective theory of gravity on cosmological scales [27, 28].

Examples of both types of lensing observables are shown in figure 1.

\subsection{The impact of systematics in shear estimation}

We now consider the main sources of systematics in estimating the lensing shears, how they are commonly described, and how they affect the measured statistics used for many cosmic shear studies in equation 2.1.

First, it is important to bear in mind that the concept of a galaxy shape is not very welldefined. Even moderate-resolution imaging reveals that galaxies do not have elliptical isophotes, but rather may have some degree of clumpiness in their light profiles and/or ellipticity gradients 

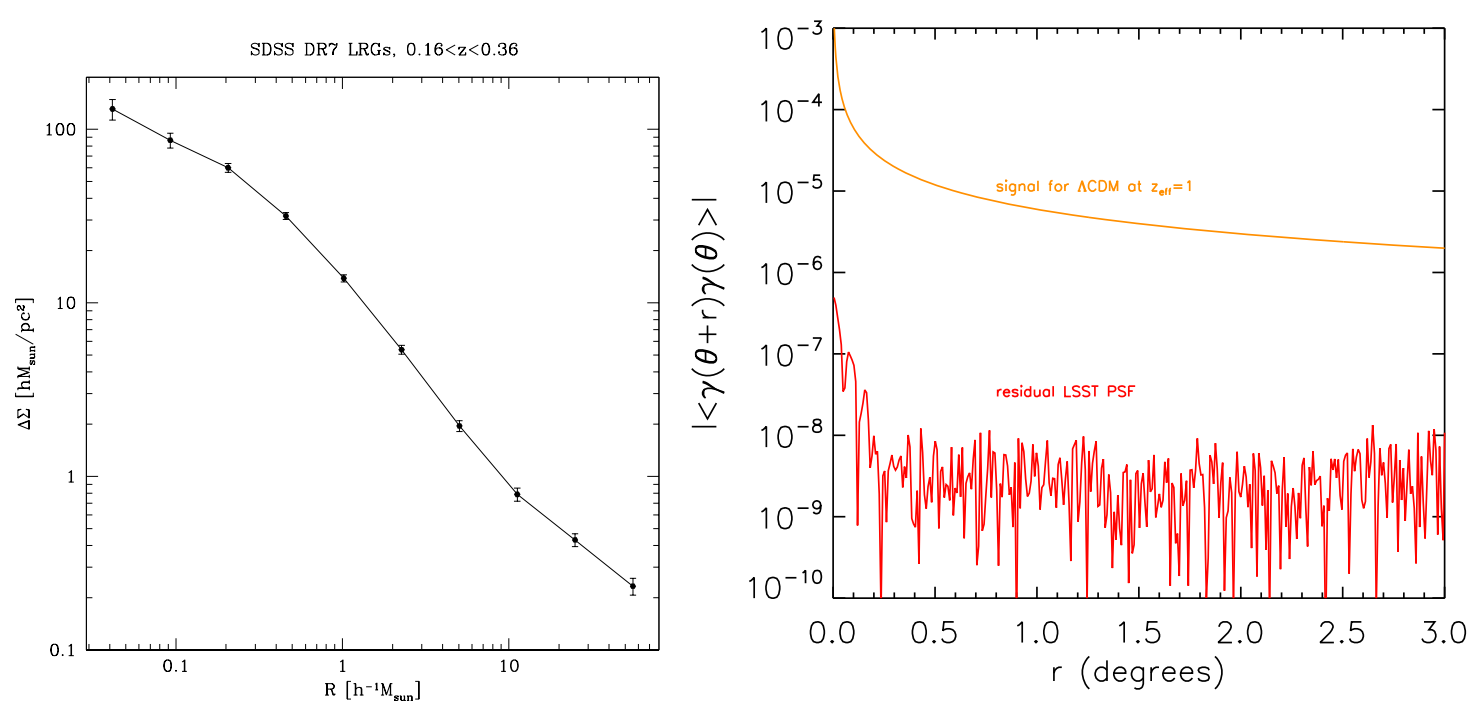

Figure 1. Left: A galaxy-galaxy lensing measurement from the Sloan Digital Sky Survey (SDSS, [26]) illustrating the projected mass profile around Luminous Red Galaxies (LRGs), including the host dark matter halo below $1 h^{-1} \mathrm{Mpc}$ and contributions from large-scale structure. At the largest scales, the signal corresponds to shears (shape distortions) of $\sim 5 \times 10^{-4}$. Right: A theoretical cosmic shear correlation function (figure 14.4 from [6]), illustrating the typical magnitude and scaling of the shear correlation function with separation, as well as an expected signal due to one particular systematic error.

(e.g. a rounder bulge component in the inner region and a more extended and flattened disk). Thus, different methods of estimating galaxy shapes will often give quite different answers for the same galaxies. Even the same method may give a different answer for a single galaxy when measuring in different passbands, due to different components of the galaxy having different spectral energy distributions. These differences are, from a weak lensing perspective, not of interest. What is important is only that a given shape estimator have a well-defined response on average to lensing shears, such that we can use the ensemble averages to measure statistics of the lensing shear field.

Images from telescopes do not directly show us the shapes of galaxies. The galaxy light profiles are modified by many effects. First and foremost is the point-spread function (PSF) of the atmosphere (for ground-based telescopes) and the telescope optics. This can be modeled as a convolution kernel that rounds galaxy shapes by a significant factor, but can have some small ellipticity that gets coherently imprinted into galaxy shapes and mimics a cosmic shear signal. There are also distortions of coordinate systems that can be treated as affine transformations rather than convolutions. Images contain defects such as cosmic rays, bleed trails, and others. Finally, the images have noise, which results in significant biases in estimates of shear [29, 30]. A schematic illustrating the image generation process is shown in figure 2. Note that the process of removing the PSF to infer the shears robustly has been the subject of focused attention from the weak lensing community for nearly two decades (with several large community-wide blind challenges [0, 11], [13, [14, [15]). Nonetheless, some issues remain to be solved, even when ignoring instrumental systematics. 


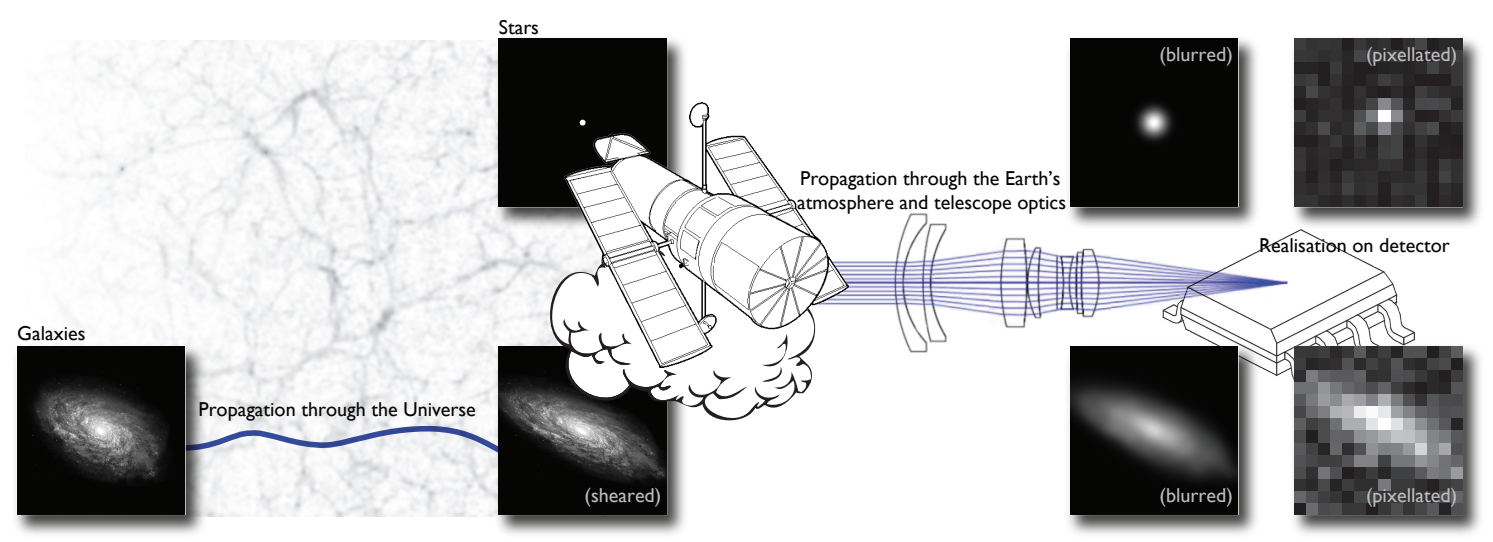

Figure 2. An illustration of the process of gravitational lensing and other effects that change the apparent shapes of galaxies in the astronomical imaging process (from [31]). Instrumental systematics are not shown, but would appear after the PSF convolution.

A common description for systematic errors in shear estimates is a linear model [32],

$$
\hat{\gamma}=(1+m) \gamma+c
$$

which can be written separately for each of the two components of the shear. Here $\gamma$ is the true lensing shear, $\hat{\gamma}$ is the estimated shear, $m$ is the multiplicative bias and $c$ the additive bias in the estimated shears. This additive bias is in general linearly proportional to the PSF ellipticity [15], though selection effects that correlate with the PSF direction might cause a deviation from this typical relationship. Ideally, $m=c=0$.

We can model galaxy-galaxy or cluster-galaxy lensing as a galaxy position $g$ vs. estimated shear $\hat{\gamma}$ cross-correlation, or (schematically)

$$
\langle g \hat{\gamma}\rangle=(1+m)\langle g \gamma\rangle+\langle g c\rangle .
$$

The first term on the right-hand side is the ideal signal with a multiplicative calibration bias determined by $m$. The second, additive term relates to the correlation between galaxy positions and additive systematics, which is typically either zero or removable through simple means [33]. Using similar notation, cosmic shear can be written as

$$
\langle\hat{\gamma} \hat{\gamma}\rangle=(1+m)^{2}\langle\gamma \gamma\rangle+2(1+m)\langle c \gamma\rangle+\langle c c\rangle .
$$

The first term on the right-hand side is the real cosmic shear with a calibration factor $(1+m)^{2} \approx$ $1+2 m$ (since typically $m \ll 1$ ). The second term would involve a correlation between additive systematics and the true shear, which may be nonzero by chance for small regions of a survey but naturally averages to zero when considering large areas. Finally, the last term is a coherent additive term in the shear field, which can typically be diagnosed using correlations between the galaxy shear estimates and star shapes [34]. Generally the scaling of this term with separation will depend on the correlation function of the PSF ellipticity or other source of additive systematics; for examples, see figure 3. Since there are clear diagnostics for additive systematics, the weak lensing 


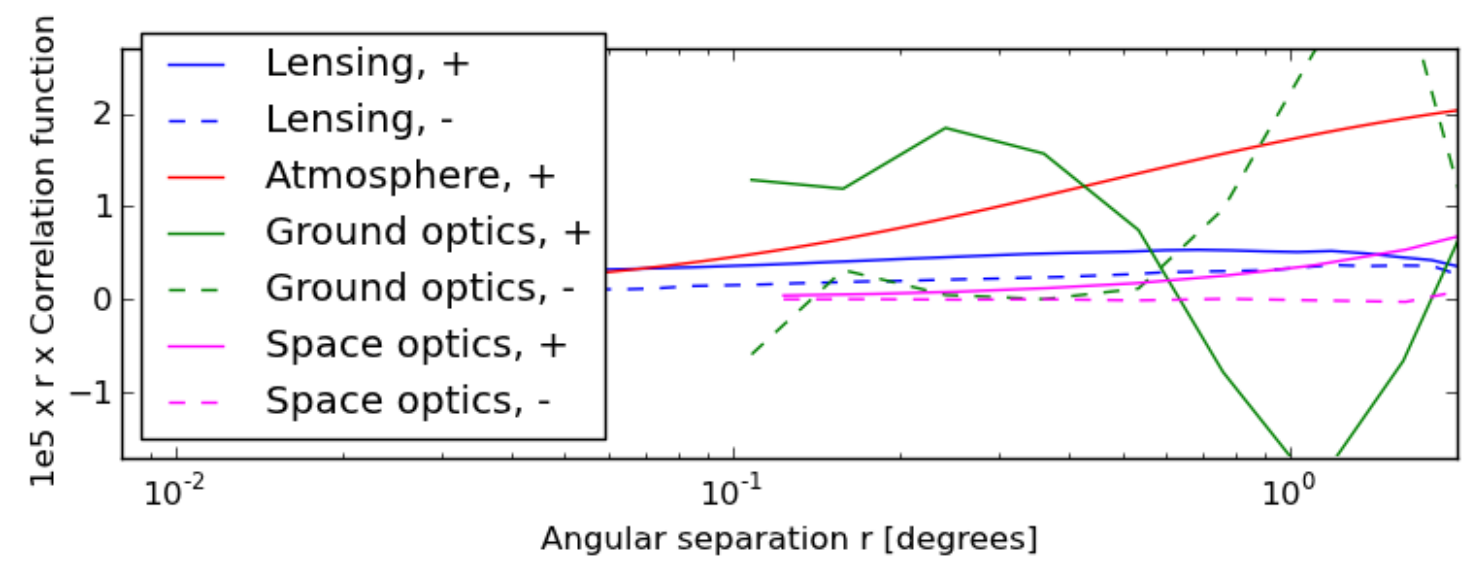

Figure 3. Lensing shear correlation functions $\xi_{ \pm}$, along with the analogous shape correlations for PSF anisotropies from several sources: typical atmospheric PSFs, and space- and ground-like optical PSFs. As shown these can have quite different scalings with separation, and at some scales they have amplitudes above the cosmic shear signal, highlighting the need for very accurate removal. From [B]].

community has primarily focused on multiplicative biases in the recent past due to the difficulty in getting an absolute calibration.

In general, this linear model for systematic errors can be used to describe basic errors in shear estimation due to the process of correcting galaxy shapes for the effects of the PSF. For other systematics, including instrumental ones, its applicability may be limited. For example, if the PSF is incorrectly modeled in a way that results in the PSF being systematically too large by the same amount at all places on the sky, then this could be modeled as a spatially constant $\langle m\rangle$ over the whole galaxy population. Real errors in the modeling of the PSF tend to have some scaledependence, and one has to consider the full spatial correlation function of those PSF modeling errors in order to really understand the impact on science. The linear model is still useful for providing basic intuition, however.

It is important to note that in general, $m=m(S / N$, size, morphology $)$ and therefore it is also effectively a function of redshift. The same is true for additive errors $(c)$. Thus, in a tomographic measurement, each redshift slice must have its own effective systematic errors estimated and accounted for, rather than treating the entire galaxy population in a monolithic way.

\section{Instrumental systematics}

Instrumental systematics include a variety of effects such as nonlinearity, charge-transfer inefficiency (most notably in the Hubble Space Telescope Advanced Camera for Surveys, [35]), tree rings and edge distortions [B6], the brighter-fatter effect [37], image defects leading to coherent selection effects (masking bias, [38]), amplifier overshoot, crosstalk, fringes, and gate diffraction. At a lower level, there are also possible chromatic instrumental effects, which would affect galaxy and star images differently due to their different spectral energy distributions. Several of these effects (tree rings, edge distortions) can be treated as part of the WCS (world coordinate system) mapping from image to world coordinates, albeit a rather complex part of the WCS. They do not necessarily 
fit cleanly into the model for systematics presented in equation 2.2. Here we discuss two of these issues in more detail.

\subsection{Brighter-fatter effect}

The brighter-fatter effect [37, 39] is a result of charge building up in pixels and repelling additional electrons (pushing them toward neighboring pixels). This makes the PSF larger for brighter objects and smaller for fainter objects. The problem for weak lensing is that bright stars (signal-to-noise ratios of $\gtrsim 50$ ) are typically used to estimate the PSF, while the galaxies used to estimate weak lensing shear are on average quite faint. Thus, their effective PSF will be smaller than the effective PSF model inferred from stars, and the PSF correction routine will systematically over-correct for the blurring effects of the PSF, resulting in overestimates of shear.

We can use a simple model for how the PSF affects galaxy shear measurements to estimate the impact of the brighter-fatter effect (if left uncorrected). Let us assume that for some particular CCD, the linear PSF size (e.g., its FWHM) will be overestimated by $1 \%$ due to the brighter-fatter effect (with $2 \%$ being the maximum effect seen in [37] using a wide range of fluxes). [40] showed that in the Gaussian approximation, the systematic bias in the inferred shear $(m=\delta \hat{\gamma} / \hat{\gamma})$ due to a misestimate of the PSF size is

$$
\frac{\delta \hat{\gamma}}{\hat{\gamma}}=\left(R_{2}^{-1}-1\right) \frac{\delta T^{(P)}}{T^{(P)}} .
$$

Here, $T^{(P)}$ is the trace of the second moment matrix of the PSF, and $\delta T^{(P)} / T^{(P)}$ is its fractional error. If the linear size of the PSF is misestimated by $1 \%$ then $\delta T^{(P)} / T^{(P)} \approx 0.02 . R_{2}$ is the galaxy "resolution factor", which is 0 for unresolved objects and approaches 1 for highly resolved galaxies. A galaxy near the resolution limit might have $R_{2} \approx 0.25$, which results in a shear calibration bias $m=0.06$. Moderately resolved galaxies $\left(R_{2} \approx 0.5\right)$ would have $m=0.02$. For context, large future surveys require that the calibration bias be reduced to the level of $m \lesssim(1-3) \times 10^{-3}$, or at least be known and reliably corrected at that level [6, 41], in order for the calibration bias to not contaminate the dark energy constraints at a level comparable to the statistical errors.

If the brighter-fatter effect has some directionality, then the PSF ellipticity for typical galaxies might likewise be misestimated. For typical methods of shear estimation, a few percent of the PSF ellipticity leaks coherently as an additive $c$ term in the galaxy shears [15]. If the PSF ellipticity error due to the brighter-fatter effect is 0.01 (coherently), then a typical shear estimation method will end up with a $c$ term of $\sim 3 \times 10^{-4}$, again slightly above the requirements for future surveys.

Clearly both the multiplicative and additive biases that may be induced by the brighter-fatter effect are larger than the requirements for upcoming surveys, so this effect must be well-modeled and removed.

\subsection{Nonlinearity}

An example of a long-known instrumental effect is CCD nonlinearity, which can be important at the high flux levels observed in the bright star images commonly used to estimate the PSF (but not for most galaxy images). CCD nonlinearity is commonly measured in the lab and then removed, making it an essentially ignored effect for most weak lensing shear studies. However, if there is some error in the nonlinearity estimation, or if it is a poorly-tracked function of time such that 
the original corrections cannot be used later on, this can cause errors in the "corrected" images of bright stars used to estimate the PSF, meaning that the PSF model used to correct the galaxy shapes is wrong.

An example of this error in practice has been seen in the SDSS. As shown in [38], there is an error in the PSF model and shear estimates in one of the six $r$-band CCDs, for which the most likely explanation is an error in the nonlinearity corrections ${ }^{5}$. The impact of this effect can be seen in figure 8 of that work, which shows a coherent systematic error in the galaxy shapes at the level of $5 \times 10^{-3}$. [26] showed that the majority of the additive systematic errors in the lensing shear in the entire SDSS area are eliminated after removing the $1 / 6$ of the data from that one CCD. While it seems unlikely that the nonlinearity for CCDs for future surveys will be misestimated in advance, if there is any change in that nonlinearity in the years needed for these surveys to be completed, then systematic errors of this type are possible.

\section{Conclusions}

Instrumental systematics can leak into weak gravitational lensing measurements in surprising ways. We have described the quantities that are measured as part of a weak lensing measurement, and gave examples for how two particular instrumental systematics can affect those measurements. In future, more work will be needed to fully characterize the various instrumental systematics that will affect surveys such as the LSST. There are, fortunately, several means for doing so. Laboratory tests can be used to understand the magnitude of the effects, which can then be included in survey image simulation software such as that for LSST (PhoSim: [42, 43]). Several instrumental effects have also been included in GalSim ${ }^{6}$ [44], an open-source image simulation software package, which can be used for more focused studies of particular instrumental effects without including all possible systematics. In either case, these simulation packages can be used to simulate images and therefore estimate the effects of these systematics on the correlation functions used to measure cosmic shear and cluster-galaxy or galaxy-galaxy lensing, and thereby estimate how the systematic errors affect inferences about dark matter and dark energy. The simulations can also be used (perhaps even more importantly) for tests of mitigation schemes.

\section{Acknowledgments}

RM is supported by the Department of Energy Early Career Award Program.

\section{References}

[1] P. Schneider, Part 3: Weak gravitational lensing, in Saas-Fee Advanced Course 33: Gravitational Lensing: Strong, Weak and Micro (G. Meylan, P. Jetzer, P. North, P. Schneider, C. S. Kochanek, and J. Wambsganss, eds.), pp. 269-451, 2006.

[2] H. Hoekstra and B. Jain, Weak Gravitational Lensing and Its Cosmological Applications, Annual Review of Nuclear and Particle Science 58 (Nov., 2008) 99-123, arXiv:0805.0139.

\footnotetext{
${ }^{5} \mathrm{R}$. Lupton, private communication.

${ }^{6}$ https://github.com/GalSim-developers/GalSim
} 
[3] R. Massey, T. Kitching, and J. Richard, The dark matter of gravitational lensing, Reports on Progress in Physics 73 (Aug., 2010) 086901-+, arXiv:1001.1739].

[4] W. Hu, Dark synergy: Gravitational lensing and the CMB, Phys. Rev. D 65 (Jan., 2002) 023003.

[5] R. Laureijs, J. Amiaux, S. Arduini, J. . Auguères, J. Brinchmann, R. Cole, M. Cropper, C. Dabin, L. Duvet, A. Ealet, and et al., Euclid Definition Study Report, ArXiv e-prints (1110.3193) (Oct., 2011) arXiv:1110.3193.

[6] LSST Science Collaborations and LSST Project, LSST Science Book, Version 2.0, ArXiv e-prints (0912.0201), http://www. Isst.org/Isst/scibook (Dec., 2009) arXiv:0912.0201].

[7] D. Spergel, N. Gehrels, J. Breckinridge, M. Donahue, A. Dressler, B. S. Gaudi, T. Greene, O. Guyon, C. Hirata, and et al., Wide-Field InfraRed Survey Telescope-Astrophysics Focused Telescope Assets WFIRST-AFTA Final Report, ArXiv e-prints (1305.5422) (May, 2013) arXiv:1305.5422.

[8] A. Albrecht, G. Bernstein, R. Cahn, W. L. Freedman, J. Hewitt, W. Hu, J. Huth, M. Kamionkowski, E. W. Kolb, L. Knox, J. C. Mather, S. Staggs, and N. B. Suntzeff, Report of the Dark Energy Task Force, ArXiv e-prints (astro-ph/0609591) (Sept., 2006) [astro-ph/0609591].

[9] C. Heymans, L. Van Waerbeke, D. Bacon, J. Berge, G. Bernstein, E. Bertin, S. Bridle, and et al., The Shear Testing Programme - I. Weak lensing analysis of simulated ground-based observations, MNRAS 368 (May, 2006) 1323-1339, [astro-ph/0506112].

[10] Z. Ma, W. Hu, and D. Huterer, Effects of Photometric Redshift Uncertainties on Weak-Lensing Tomography, ApJ 636 (Jan., 2006) 21-29, astro-ph/0506614].

[11] R. Massey, C. Heymans, J. Bergé, G. Bernstein, S. Bridle, D. Clowe, H. Dahle, R. Ellis, and et al., The Shear Testing Programme 2: Factors affecting high-precision weak-lensing analyses, MNRAS 376 (Mar., 2007) 13-38, astro-ph/0].

[12] G. Bernstein and D. Huterer, Catastrophic photometric redshift errors: weak-lensing survey requirements, MNRAS 401 (Jan., 2010) 1399-1408, [arXiv:0902.2782].

[13] S. Bridle, S. T. Balan, M. Bethge, M. Gentile, S. Harmeling, C. Heymans, M. Hirsch, R. Hosseini, M. Jarvis, D. Kirk, and et al., Results of the GREAT08 Challenge: an image analysis competition for cosmological lensing, MNRAS 405 (July, 2010) 2044-2061, arXiv:0908.0945.

[14] T. D. Kitching, S. T. Balan, S. Bridle, N. Cantale, F. Courbin, T. Eifler, M. Gentile, M. S. S. Gill, S. Harmeling, C. Heymans, M. Hirsch, and et al., Image analysis for cosmology: results from the GREAT10 Galaxy Challenge, MNRAS 423 (July, 2012) 3163-3208, [arXiv:1202.5254].

[15] R. Mandelbaum, B. Rowe, R. Armstrong, D. Bard, E. Bertin, J. Bosch, D. Boutigny, F. Courbin, W. A. Dawson, A. Donnarumma, I. Fenech Conti, R. Gavazzi, M. Gentile, M. Gill, D. W. Hogg, E. M. Huff, M. J. Jee, T. Kacprzak, M. Kilbinger, T. Kuntzer, D. Lang, W. Luo, M. C. March, P. J. Marshall, J. E. Meyers, L. Miller, H. Miyatake, R. Nakajima, F. M. Ngole Mboula, G. Nurbaeva, Y. Okura, S. Paulin-Henriksson, J. Rhodes, M. D. Schneider, H. Shan, E. S. Sheldon, M. Simet, J.-L. Starck, F. Sureau, M. Tewes, K. Zarb Adami, J. Zhang, and J. Zuntz, GREAT3 results I: systematic errors in shear estimation and the impact of real galaxy morphology, ArXiv e-prints (Dec., 2014) arXiv:1412.1825.

[16] I. Mohammed and U. Seljak, Analytic model for the matter power spectrum, its covariance matrix and baryonic effects, MNRAS 445 (Dec., 2014) 3382-3400, [arXiv:1407.0060.

[17] M. A. Troxel and M. Ishak, The Intrinsic Alignment of Galaxies and its Impact on Weak Gravitational Lensing in an Era of Precision Cosmology, ArXiv e-prints (July, 2014) arXiv:1407.6990. 
[18] G. M. Bernstein and R. Armstrong, Bayesian lensing shear measurement, MNRAS 438 (Feb., 2014) 1880-1893, arXiv:1304.1843.

[19] G. M. Bernstein and M. Jarvis, Shapes and Shears, Stars and Smears: Optimal Measurements for Weak Lensing, AJ 123 (Feb., 2002) 583-618, [astro-ph/0107431].

[20] C. Heymans, E. Grocutt, A. Heavens, M. Kilbinger, T. D. Kitching, F. Simpson, J. Benjamin, T. Erben, H. Hildebrandt, H. Hoekstra, and et al., CFHTLenS tomographic weak lensing cosmological parameter constraints: Mitigating the impact of intrinsic galaxy alignments, MNRAS 432 (July, 2013) 2433-2453, arXiv:1303.1808].

[21] H. Lin, S. Dodelson, H.-J. Seo, M. Soares-Santos, J. Annis, J. Hao, D. Johnston, J. M. Kubo, R. R. R. Reis, and M. Simet, The SDSS Co-add: Cosmic Shear Measurement, ApJ 761 (Dec., 2012) 15, arXiv:1111.6622.

[22] M. J. Jee, J. A. Tyson, M. D. Schneider, D. Wittman, S. Schmidt, and S. Hilbert, Cosmic Shear Results from the Deep Lens Survey. I. Joint Constraints on $\Omega_{M}$ and $\sigma_{8}$ with a Two-dimensional Analysis, ApJ 765 (Mar., 2013) 74, arXiv:1210.2732].

[23] E. M. Huff, T. Eifler, C. M. Hirata, R. Mandelbaum, D. Schlegel, and U. Seljak, Seeing in the dark II. Cosmic shear in the Sloan Digital Sky Survey, MNRAS 440 (May, 2014) 1322-1344.

[24] J. Coupon, S. Arnouts, L. van Waerbeke, T. Moutard, O. Ilbert, E. van Uitert, T. Erben, B. Garilli, L. Guzzo, C. Heymans, H. Hildebrandt, H. Hoekstra, M. Kilbinger, T. Kitching, Y. Mellier, L. Miller, M. Scodeggio, C. Bonnett, E. Branchini, I. Davidzon, G. De Lucia, A. Fritz, L. Fu, P. Hudelot, M. J. Hudson, K. Kuijken, A. Leauthaud, O. Le Fèvre, H. J. McCracken, L. Moscardini, B. T. P. Rowe, T. Schrabback, E. Semboloni, and M. Velander, The galaxy-halo connection from a joint lensing, clustering and abundance analysis in the CFHTLenS/VIPERS field, ArXiv e-prints (Feb., 2015) arXiv:1502.02867.

[25] M. Simet, N. Battaglia, R. Mandelbaum, and U. Seljak, Weak lensing calibration of mass bias in the RBC X-ray galaxy cluster catalog, ArXiv e-prints (Feb., 2015) [arXiv:1502.01024].

[26] R. Mandelbaum, A. Slosar, T. Baldauf, U. Seljak, C. M. Hirata, R. Nakajima, R. Reyes, and R. E. Smith, Cosmological parameter constraints from galaxy-galaxy lensing and galaxy clustering with the SDSS DR7, MNRAS 432 (June, 2013) 1544-1575, [arXiv:1207.1120].

[27] R. Reyes, R. Mandelbaum, U. Seljak, T. Baldauf, J. E. Gunn, L. Lombriser, and R. E. Smith, Confirmation of general relativity on large scales from weak lensing and galaxy velocities, Nature $\mathbf{4 6 4}$ (Mar., 2010) 256-258, arXiv:1003.2185].

[28] P. Zhang, M. Liguori, R. Bean, and S. Dodelson, Probing Gravity at Cosmological Scales by Measurements which Test the Relationship between Gravitational Lensing and Matter Overdensity, Physical Review Letters 99 (Oct., 2007) 141302, [arXiv:0704.1932].

[29] T. Kacprzak, J. Zuntz, B. Rowe, S. Bridle, A. Refregier, A. Amara, L. Voigt, and M. Hirsch, Measurement and calibration of noise bias in weak lensing galaxy shape estimation, MNRAS $\mathbf{4 2 7}$ (Dec., 2012) 2711-2722, [arXiv:1203.5049].

[30] A. Refregier, T. Kacprzak, A. Amara, S. Bridle, and B. Rowe, Noise bias in weak lensing shape measurements, MNRAS 425 (Sept., 2012) 1951-1957, [arXiv:1203.5050.

[31] R. Mandelbaum, B. Rowe, J. Bosch, C. Chang, F. Courbin, M. Gill, M. Jarvis, A. Kannawadi, T. Kacprzak, C. Lackner, and et al., The Third Gravitational Lensing Accuracy Testing (GREAT3) Challenge Handbook, ApJS 212 (May, 2014) 5, [arXiv:1308.4982]. 
[32] D. Huterer, M. Takada, G. Bernstein, and B. Jain, Systematic errors in future weak-lensing surveys: requirements and prospects for self-calibration, MNRAS 366 (Feb., 2006) 101-114, astro-ph/0506030.

[33] R. Mandelbaum, C. M. Hirata, U. Seljak, J. Guzik, N. Padmanabhan, C. Blake, M. R. Blanton, R. Lupton, and J. Brinkmann, Systematic errors in weak lensing: application to SDSS galaxy-galaxy weak lensing, MNRAS 361 (Aug., 2005) 1287-1322, astro-ph/0501201].

[34] C. Heymans, L. Van Waerbeke, L. Miller, T. Erben, H. Hildebrandt, H. Hoekstra, T. D. Kitching, Y. Mellier, P. Simon, C. Bonnett, J. Coupon, L. Fu, J. Harnois Déraps, M. J. Hudson, M. Kilbinger, K. Kuijken, B. Rowe, T. Schrabback, E. Semboloni, E. van Uitert, S. Vafaei, and M. Velander, CFHTLenS: the Canada-France-Hawaii Telescope Lensing Survey, MNRAS 427 (Nov., 2012) 146-166, arXiv:1210.0032].

[35] R. Massey, C. Stoughton, A. Leauthaud, J. Rhodes, A. Koekemoer, R. Ellis, and E. Shaghoulian, Pixel-based correction for Charge Transfer Inefficiency in the Hubble Space Telescope Advanced Camera for Surveys, MNRAS 401 (Jan., 2010) 371-384, arXiv:0909.0507].

[36] A. A. Plazas, G. M. Bernstein, and E. S. Sheldon, On-Sky Measurements of the Transverse Electric Fields' Effects in the Dark Energy Camera CCDs, PASP 126 (Sept., 2014) 750-760.

[37] P. Antilogus, P. Astier, P. Doherty, A. Guyonnet, and N. Regnault, The brighter-fatter effect and pixel correlations in CCD sensors, Journal of Instrumentation 9 (Mar., 2014) C3048, arXiv: 1402.0725 .

[38] E. M. Huff, C. M. Hirata, R. Mandelbaum, D. Schlegel, U. Seljak, and R. H. Lupton, Seeing in the dark - I. Multi-epoch alchemy, MNRAS 440 (May, 2014) 1296-1321.

[39] A. Guyonnet, P. Astier, P. Antilogus, N. Regnault, and P. Doherty, Evidence for self-interaction of charge distribution in charge-coupled devices, A\&A 575 (Mar., 2015) A41, arXiv:1501.01577.

[40] C. M. Hirata, R. Mandelbaum, U. Seljak, J. Guzik, N. Padmanabhan, C. Blake, J. Brinkmann, T. Budávari, A. Connolly, I. Csabai, R. Scranton, and A. S. Szalay, Galaxy-galaxy weak lensing in the Sloan Digital Sky Survey: intrinsic alignments and shear calibration errors, MNRAS 353 (Sept., 2004) 529-549, astro-ph/0].

[41] R. Massey, H. Hoekstra, T. Kitching, J. Rhodes, M. Cropper, J. Amiaux, D. Harvey, Y. Mellier, M. Meneghetti, L. Miller, S. Paulin-Henriksson, S. Pires, R. Scaramella, and T. Schrabback, Origins of weak lensing systematics, and requirements on future instrumentation (or knowledge of instrumentation), MNRAS 429 (Feb., 2013) 661-678, arXiv:1210.7690].

[42] A. J. Connolly, J. Peterson, J. G. Jernigan, R. Abel, J. Bankert, C. Chang, C. F. Claver, R. Gibson, D. K. Gilmore, E. Grace, R. L. Jones, Z. Ivezic, J. Jee, M. Juric, S. M. Kahn, V. L. Krabbendam, S. Krughoff, S. Lorenz, J. Pizagno, A. Rasmussen, N. Todd, J. A. Tyson, and M. Young, Simulating the LSST system, in Society of Photo-Optical Instrumentation Engineers (SPIE) Conference Series, vol. 7738 of Society of Photo-Optical Instrumentation Engineers (SPIE) Conference Series, p. 1, July, 2010.

[43] J. R. Peterson and J. G. Jernigan, "phoSim: Photon Simulator.” Astrophysics Source Code Library, July, 2013.

[44] B. Rowe, M. Jarvis, R. Mandelbaum, G. M. Bernstein, J. Bosch, M. Simet, J. E. Meyers, T. Kacprzak, R. Nakajima, J. Zuntz, H. Miyatake, J. P. Dietrich, R. Armstrong, P. Melchior, and M. S. S. Gill, GalSim: The modular galaxy image simulation toolkit, ArXiv e-prints (1407.7676) (July, 2014). 